This item was submitted to Loughborough's Research Repository by the author.

Items in Figshare are protected by copyright, with all rights reserved, unless otherwise indicated.

\title{
3D-printed millimeter wave lens antenna
}

PLEASE CITE THE PUBLISHED VERSION

https://doi.org/10.1109/GSMM.2017.7970303

\section{PUBLISHER}

(C) Institute of Electrical and Electronics Engineers (IEEE)

\section{VERSION}

AM (Accepted Manuscript)

\section{LICENCE}

CC BY-NC-ND 4.0

\section{REPOSITORY RECORD}

Arya, Ravi, Shiyu Zhang, J.C. Vardaxoglou, W.G. Whittow, and Raj Mittra. 2019. "3d-printed Millimeter Wave Lens Antenna". figshare. https://hdl.handle.net/2134/28036. 


\title{
3D-Printed Millimeter Wave Lens Antenna
}

\author{
Ravi Kumar Arya ${ }^{1}$, Shiyu Zhang ${ }^{2}$, Yiannis Vardaxoglou ${ }^{2}$, Will Whittow ${ }^{2}$ and Raj Mittra ${ }^{3}$ \\ ${ }^{1}$ EMC Laboratory, The Pennsylvania State University, USA \\ ${ }^{2}$ Wolfson School of Mechanical, Electrical and Manufacturing Engineering, Loughborough University, UK \\ ${ }^{3}$ University of Central Florida, USA and KAU, Saudi Arabia \\ rka5048@psu.edu
}

\begin{abstract}
In this work, we present a flat lens design using the Dial-a-Dielectric (DaD) and 3D-printing technique to realize the materials that are not available off-the-shelf. We design the proposed flat lens and compare its performance with that of the ray-optics (RO)-based lens. We find from the results that both designs show comparable performance.
\end{abstract}

\section{INTRODUCTION}

The design of flat lens antennas have been discussed in several recent publications [1] - [7]. Such a lens antenna can be designed to have a high gain and wide bandwidth and still maintain its low-profile characteristics.

The lens can be designed as a collection of concentric rings, whose permittivity values can be determined by using the ray optics approach so that the path lengths of the rays passing through the lens are all equal. But, the material parameters that this type of design calls for may not be readily available commercially off-the-shelf. To address this issue, we propose to design these needed materials using a combination of $\mathrm{DaD}$ (Dial-a-Dielectric) or 3D-printing methods [8]-[9].

\section{DESIGN}

We design the RO lens in the form of concentric rings, with variable permittivity values, as shown in Fig. 11. The desired permittivity values are calculated by enforcing equation 1 . which imposes the condition that the rays from the different rings of the lens collimate at focal point with equal path lengths [10].

$$
\epsilon_{n}=\left[\frac{h \sqrt{\epsilon_{1}}+F-\sqrt{x^{2}+F^{2}}}{h}\right]^{2}
$$

The design specifications of the lens (see Fig. 1 ) are: frequncy range $f=30-35 \mathrm{GHz}$; focal length $F=150 \mathrm{~mm}$; thickness $h=13.08 \mathrm{~mm}$. The diameter $D(120 \mathrm{~mm})$ and, hence, the number of rings are chosen to satisfy the gain requirements. The number of rings is chosen to be 6 and each ring has a width of $10 \mathrm{~mm}$. The desired permittivity values that are needed to satisfy the path length condition for these rings are shown in Table 1 and have been obtained by using equation 1 .

A quick search of available materials reveals that not all of these materials are commercially available, off-the-shelf; consequently, we use the Dial-a-Dielectric (DaD) approach, in conjunction with the 3D-printing technique to realize these materials. For this design, we use the 3D-material with $\epsilon=$

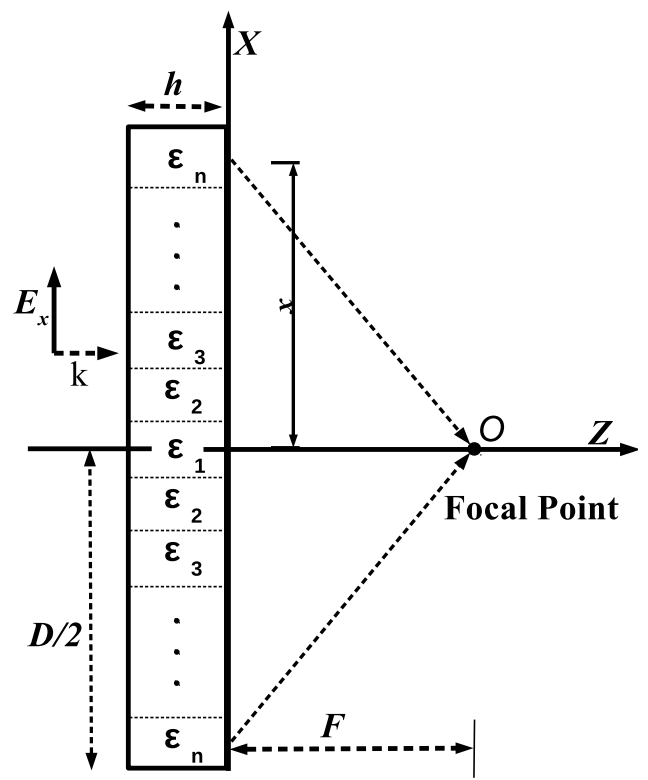

Fig. 1: Lens design principle

2.72. So, if the permittivity needed for a particular ring exceeds 2.72 , we combine the $\mathrm{DaD}$ approach with the $3 \mathrm{D}$ printing to realize it. Looking at Table [1. we can see that the rings 1-3 require a combination of the $\mathrm{DaD}$ technique with $3 \mathrm{D}$ printing, while the 3D printing alone is adequate for the rings 4-6. In the $\mathrm{DaD}$ approach we modify the permittivities of the COTS materials, by covering these materials with metallic patches while in the 3D-printing technique, we insert air voids in the 3D-printing material to realize the required permittivity. We have found that neither $\mathrm{DaD}$ nor the 3D-printing approaches [8]-[9] lead to material realizations that are lossy, dispersive and narrowband, as they would be if we had used resonant metamaterials instead.

For comparison, we design two lenses: one (uniform dielectric lens, RO lens) with parameters shown in Table I and the other (proposed lens) whose parameters are presented in Fig. 2 and Table II The local periodicity of the patches is chosen to be $2 \mathrm{~mm} \times 2 \mathrm{~mm}$ for this design. For both lenses, full wave simulations are run in HFSS. 
TABLE I: Material Parameters of Uniform Dielectrics Lens.

\begin{tabular}{|c|c|c|c|c|c|}
\hline$\epsilon_{1}$ & $\epsilon_{2}$ & $\epsilon_{3}$ & $\epsilon_{4}$ & $\epsilon_{5}$ & $\epsilon_{6}$ \\
\hline 3.46 & 3.25 & 2.90 & 2.41 & 1.84 & 1.24 \\
\hline
\end{tabular}

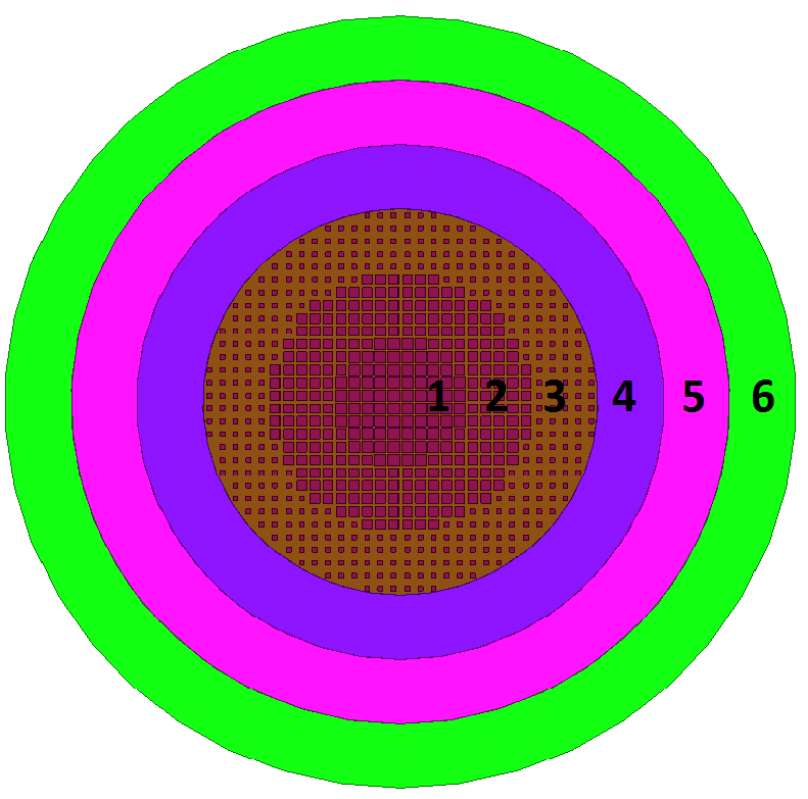

(a) Top view

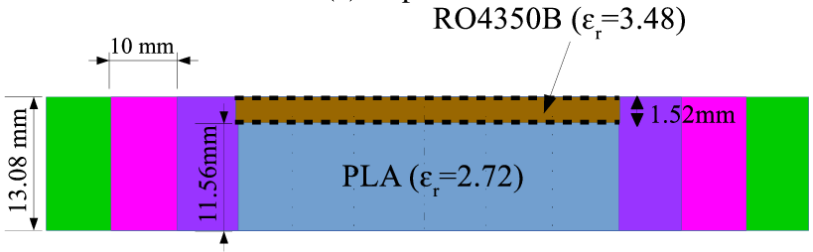

(b) Cross-sectional view

Fig. 2: Proposed Lens

\section{RESULTS}

Fig. 3 shows the electric field distribution along the axis of the RO lens when a plane wave is incident upon the lens from the left. Fig.4 shows that the electric field converges at the focal point $(O)$. Similarly in Fig. 4 we compare the electric field magnitudes along the axis for both lenses. We note from this figure that the field magnitudes at the focal point are comparable for both lenses. It is important to point out that the materials for the RO lens aren't available and we had to artificially synthesize them in the proposed lens.

Fig. 5 shows the gain, defined as $20 \log 10\left(\left|E_{f}\right| /\left|E_{i}\right|\right)$, where $E_{f}$ is electric field at the focal point (' $O$ ') and $E_{i}$ is the electric field of the incident plane wave (here, $E_{i}=1 \mathrm{~V} / \mathrm{m}$ for simulation). The gain values are within $0.6 \mathrm{~dB}$ of each other.

\section{CONCLusions}

Two different flat lens designs have been presented in this work. The first of these, namely the RO Lens, is based on the use of uniform dielectrics that are not commercially available,
TABLE II: Patch Sizes for Proposed Lens

\begin{tabular}{|c|c|}
\hline Ring No. & Patch size \\
\hline 1 & $1.46 \mathrm{~mm} \times 1.46 \mathrm{~mm}$ \\
\hline 2 & $1.35 \mathrm{~mm} \times 1.35 \mathrm{~mm}$ \\
\hline 3 & $0.9 \mathrm{~mm} \times 0.9 \mathrm{~mm}$ \\
\hline 4 & N/A \\
\hline 5 & N/A \\
\hline 6 & N/A \\
\hline
\end{tabular}

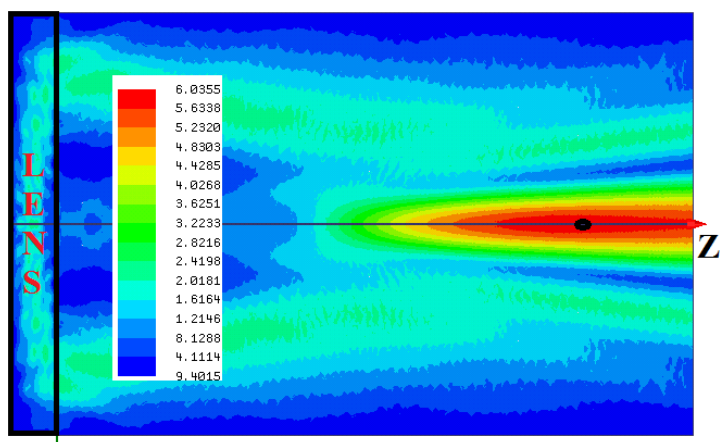

(a) Magnitude

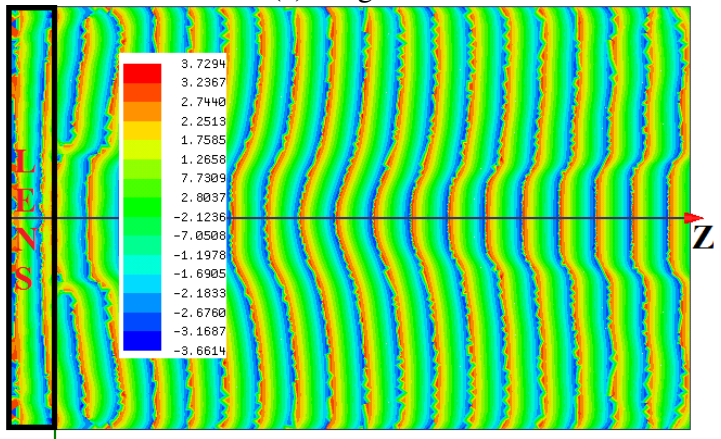

(b) Phase

Fig. 3: Electric field distribution at $30 \mathrm{GHz}$

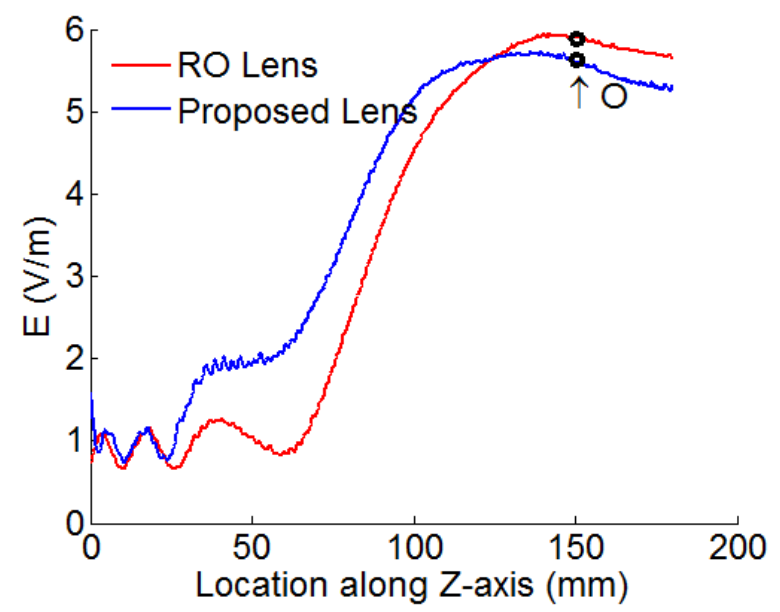

Fig. 4: Electric field distribution along axial direction at $30 \mathrm{GHz}$ 


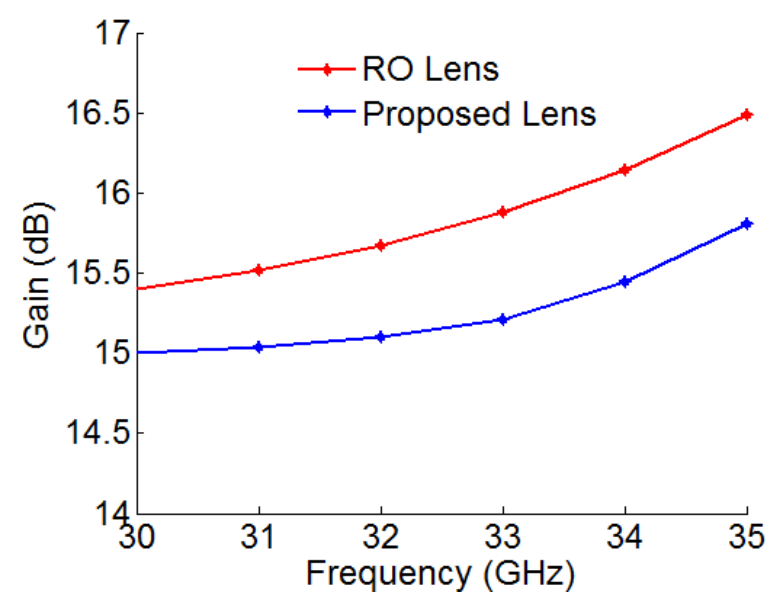

Fig. 5: Simulated gain of lenses

and is included here for comparison. The second lens is designed using the $\mathrm{DaD}$, and its performance is comparable to that of the RO lens, which requires permittivities not available off-the-shelf.

\section{REFERENCES}

[1] A. Bisognin et al., "3D printed plastic $60 \mathrm{GHz}$ lens: Enabling innovative millimeter wave antenna solution and system," 2014 IEEE MTT-S International Microwave Symposium, Tampa, FL, 2014, pp. 1-4.

[2] E. L. Holzman, "A highly compact $60-\mathrm{GHz}$ lens-corrected conical horn antenna," IEEE Antennas and Wireless Propagation Letters, vol. 3, no. 1, pp. 280-282, Dec. 2004

[3] P. Nayeri et al., "3D Printed Dielectric Reflectarrays: Low-Cost HighGain Antennas at Sub-Millimeter Waves," in IEEE Transactions on Antennas and Propagation, vol. 62, no. 4, pp. 2000-2008, April 2014.

[4] M.K. Taher Al-Nuaimi, Wei Hong, "Fabrication and experimental validation of high gain lens antenna for $71-86 \mathrm{GHz}$ band," IEEE International in Wireless Symposium (IWS), pp. 1-4, March 30 - April 1, 2015

[5] R. Yang, W. Tang and Y. Hao, "A broadband zone plate lens from transformation optics," Opt. Express, vol. 19, no. 13, pp. 12348-12355, 2011.

[6] Y. Zhang, R. Mittra and W. Hong, "A zoned two-layer flat lens design," International Workshop on Antenna Technology (iWAT), pp. 412-415, March 7-9, 2011

[7] E. Erfani, M. Niroo-Jazi and S. Tatu, "A High-Gain Broadband Gradient Refractive Index Metasurface Lens Antenna," in IEEE Transactions on Antennas and Propagation, vol. 64, no. 5, pp. 1968-1973, May 2016.

[8] R. K. Arya, S. Pandey, and R. Mittra, "Flat lens design using artificially engineered materials," Progress In Electromagnetics Research C, Vol. 64, 71-78, 2016.

[9] S. Zhang, R. K. Arya, S. Pandey, Y. Vardaxoglou, W. Whittow and R. Mittra, "3D-printed planar graded index lenses," IET Microwaves, Antennas \& Propagation, vol. 10, no. 13, pp. 1411-1419, 2016.

[10] A. O. Pinchuk and G. C. Schatz, "Metamaterials with gradient negative index of refraction," J. Opt. Soc. Am. A 24, A39-A44 (2007). 